\title{
A simulation study of excitation coil design in single-sided MPI scanner for human body application
}

\author{
Nurmiza Othman ${ }^{1}$, Muhamad Fikri Shahkhirin Birahim ${ }^{2}$, Wan Nurshazwani Wan Zakaria ${ }^{3}$, Mohd \\ Razali Md Tomari ${ }^{4}$, Md Nor Ramdon Baharom ${ }^{5}$, Luqman Hakim ${ }^{6}$ \\ ${ }^{1,2}$ Department of Electronic Engineering, Faculty of Electrical and Electronic Engineering, Universiti Tun Hussein Onn \\ Malaysia, Malaysia \\ ${ }^{3,4}$ Department Department of Mechatronic and Robotic Engineering, Faculty of Electrical and Electronic Engineering, \\ Universiti Tun Hussein Onn Malaysia, Malaysia \\ ${ }^{5,6}$ Department Department of Electrical Power Engineering, Faculty of Electrical and Electronic Engineering, Universiti \\ Tun Hussein Onn Malaysia, Malaysia
}

\begin{tabular}{l} 
Article Info \\
\hline Article history: \\
Received Mar 29, 2019 \\
Revised May 26, 2019 \\
Accepted Jun 14, 2019 \\
\hline
\end{tabular}

Keywords:

Magnetic coil

Magnetic particle imaging

Medical imaging

Sentinel lymph node

Superparamagnetic iron oxide

Nanoparticles

\begin{abstract}
Magnetic particle imaging (MPI), a tomographic imaging method has been introduced for 3D imaging of human body with some potential applications such as magnetic hyperthermia and cancer imaging. It involves three important elements: tracer development using magnetic nanoparticles (MNPs), hardware realization (scanner using excitation and pickup coils), and image reconstruction optimization. Their combination will produce a high quality of image taken from any biological tissue in the human body based on the secondary magnetic field signal from the magnetized MNPs that are injected into human body. A homogeneous and adequate magnetic field strength from an excitation coil is needed to enhance the quality of the secondary signal. However, the complex surface topography of human body and physical properties of an excitation coil influence the strength and the homogeneity of the magnetic field generation at the MNPs. Therefore, this work focused on finding alternative design of excitation coil used in single sided MPI to produce up to $2 m T$ with high homogeneity of field distribution in the MNPs at the varied depth of 10 to $30 \mathrm{~mm}$ under the excitation coil. We proposed several designs with variation in physical properties and coil arrangement based on simulation study carried out by using Ansys Maxwell.
\end{abstract}

Copyright $\left({ }_{0} 2019\right.$ Institute of Advanced Engineering and Science. All rights reserved.

\section{Corresponding Author:}

Nurmiza Othman,

Department of Electronic Engineering,

Faculty of Electrical and Electronic Engineering,

Universiti Tun Hussein Onn Malaysia, 86400 Parit Raja, Johor, Malaysia.

Email: nurmiza@uthm.edu.my

\section{INTRODUCTION}

Magnetic nanoparticles (MNPs) show great potential in environmental, biomedical, and clinical applications due to their many remarkable properties [1]. For instance, magnetic tracers can be obtained when MNPs are coated by some polymers and are likely may offer some attractive possibilities in biomedical applications such as cell labeling, magnetic hyperthermia, drug delivery, immunoassay, and magnetic particle imaging (MPI) [2-4]. Figure 1 shows a schematic drawing of a spherical MNP consists of a magnetic core with biocompatible coating for biomedical application. The magnetic properties of MNPs can be easily controlled using external field excitation. By exploiting their suparamagnetic behavior under magnetic field exposure, they can be used to extend novel imaging modality known as MPI and lead to a significant development for the future of three-dimensional (3D) magnetic bioimaging of the human body [5]. 
Since its discovery in early 2000s, MPI as a tomographic imaging method has experienced a rapid development with some potential applications in cardio imaging and sentinel lymph node imaging for early stage cancer diagnosis. The invention of an MPI system involves three important elements: tracer development using magnetic nanoparticles (MNPs), hardware realization (scanner using excitation and pickup coils), and image reconstruction optimization [6-10]. Figure 2 shows a representative model of an MPI system proposed previously that can be used for sentinel lymph node imaging. Details on the experiment setup and MNP sample properties were already reported elsewhere [9]. An MPI image can be obtained after the reconstruction process of the secondary magnetic field signal detected from the distribution of MNPs that are initially injected and then accumulated at certain depth in the human body (distance across $z$ direction imitates distance between MNPs and the human body surface). The secondary signal is generated due to response of the MNPs under externally applied magnetic field from the excitation coil and can be detected by a very sensitive gradiometer pickup coil from outside of the body surface.

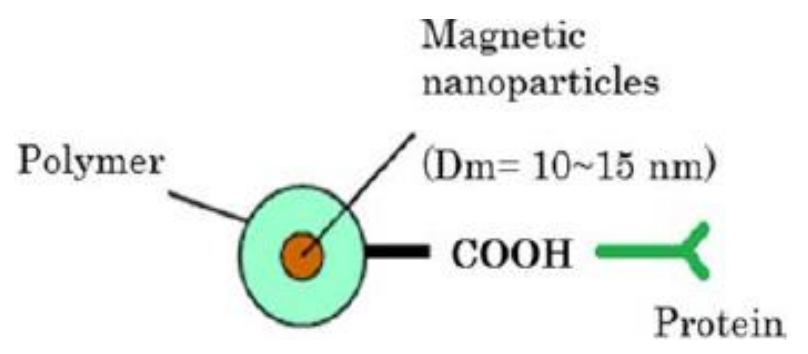

Figure 1. Schematic drawing of a spherical MNP consists of a magnetic core with biocompatible coating for biomedical applications

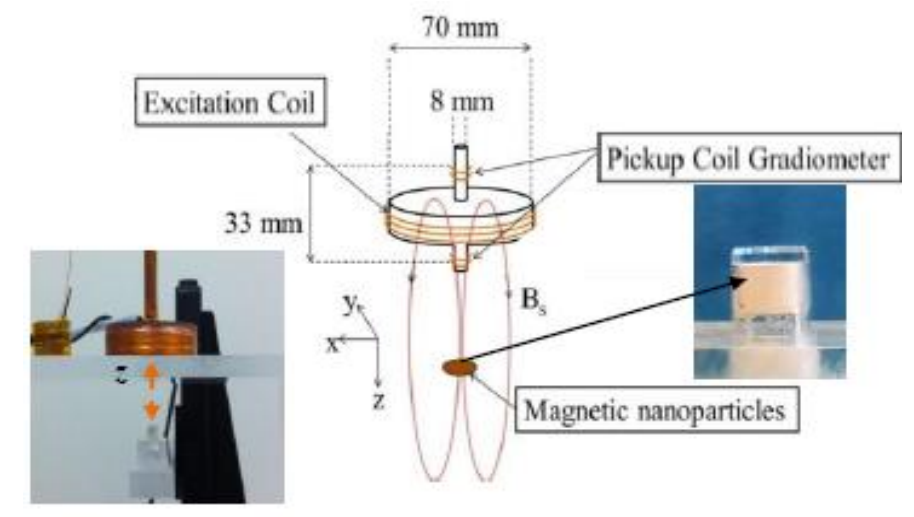

Figure 2. Physical arrangement of excitation coil and pickup coil together with the MNPs position [9]

The magnetic response is known as superparamagnetic behavior of MNPs and can be very well explained by the Langevin function as shown in (1) [11]. Magnetic moment, $m$ in each of MNPs randomly rotates owing to the thermal noise when there is no external magnetic field, $H$ added to the MNPs. Accordingly, the direction of the magnetic moment becomes random, and the mean value of the magnetic moment of the MNPs becomes zero, hence, no secondary signal from the MNPs will be produced. However, when MNPs are magnetized under certain value of $H$, the magnetic moments in the MNPs tend to align in the direction of the $H$ and produce the mean magnetic moment of MNPs, $<m>$ in the direction of $H$. $L$ indicates the Langevin function, $k \mathrm{~B}$ is the Boltzmann constant, and $T$ is the absolute temperature.

$$
\frac{<m>}{m}=L\left(\frac{m H}{k_{B} T}\right)=\operatorname{coth} \frac{m H}{k_{B} T}-\frac{k_{B} T}{m H}
$$

When an external excitation field consisting of the DC bias field $H_{\mathrm{DC}}$ and the AC field $H_{\mathrm{DC}}$ is applied to the MNPs, (1) can be rewritten as (2): 


$$
\frac{<m>}{m}=\operatorname{coth}\left[\frac{H_{D C}+\sqrt{2} H_{A C} \cos (w t)}{k_{B} T / m}\right]-\frac{k_{B} T / m}{H_{D C}+\sqrt{2} H_{A C} \cos (w t)}
$$

Finally, $n$-th harmonic signal from the MNPs can be obtained byexpanding the right-hand side of (2) into a Fourier series as shown in (3):

$$
\frac{\langle m\rangle_{n}}{m}=2 w \int_{0}^{1 / w} L\left(\frac{H_{D C}+\sqrt{2} H_{A C} \cos (w t)}{k_{B} T / m}\right) \cos (n w t) d t
$$

This $n$-th harmonic signal is very cruicial in MPI system to appear as the secondary signal that will be sensed by the gradiometer pickup coil before reconstructed to an MPI image. Therefore, in addition to develop high sensitivity detection hardware and an excellent image reconstruction method, it is very cruicial to have MNPs that can produce strong harmonic signal in order to assure the image quality in MPI. Work related to the harmonic signal characterization on several MNPs used in MPI suggested that since MPI system utilizes harmonic signals, it is important to select MNPs that can generate strong harmonic signals [12-15]. Furthermore, the contribution of $H$ in (1)-(3) proves that an adequate strength and homogeneous magnetic field generated from an excitation coil is very crucial to enhance the level of that secondary signal. The combination of these elements will produce a high quality of 3D image taken from any biological tissue in the human body using the MPI scanner. The standard MPI system that was introduced in earlier stages was set up as a cave-shaped system and a single-sided scanner where imaging takes place when MNPs be recorded at the field of view lies in between a symmetric coil configuration [5]. However, the size of specimens to be fixed into the scanning area is limited due to the limited size of the cave itself. Later, a single-sided MPI scanner was introduced in 2009 where imaging of specimen of interest simply can be done just from one side [16-17]. Hence, the problem of the specimen fitting into the cave-shaped scanner no longer exists.

Unfortunately, there are other limitations that may affect the efficiency of an MPI scanner to produce a good image. First, the complex surface topography of human body makes it harder for the scanning process to be done regardless to the single-sided MPI scanner which is said much simpler than the cave-shaped system. This will affect the reconstruction image quality to obtain the 3D information of human body. Furthermore, physical properties of an excitation coil also influence the strength and the homogeneity of the magnetic field generation at the MNPs trapped under the body surface [18]. Due to the design, size and material used for the available excitation coil, the magnetic field strength be sensed at the MNPs located under the body surfaces is weak and inhomogeneous then causes the secondary signal from the magnetized MNPs decrease. Thus far, the excitation coil which has been installed in the existing MPI system is either bulky in size or must be connected to other electronic components such as resonant circuit. It is to support the maximum level of current to be supplied to the excitation coil at $\mathrm{KHz}$ level frequency with least impedance loss in the system and MNPs can be excited maximally from the excitation coil. The bulkiness and connection to extra electronic components should be avoided so that the simplest single-sided MPI scanner can be developed and used for the scanning of the complex human body surface [19-24].

Therefore, this work focused on finding alternative design of the excitation coil in a single-sided MPI scanner to produce up to $2 \mathrm{mT}$ with high homogeneity of magnetic field distribution in the MNPs that are positioned at the varied depth of $10 \mathrm{~mm}$ to $30 \mathrm{~mm}$ under the excitation coil. Design of excitation coil was carried out and simulated by using an electromagnetic field simulation software, Ansys Maxwell. Ansys Maxwell is high performance software that uses finite element analysis (FEA) to solve magneto static, electric, eddy current and many transient problems. Ansys Maxwell solves the electromagnetic problems by solving Maxwell's equation in the software calculation process [25]. Physical properties such as type of material, diameter, and shape were varied and modeled to find the suitable parameter of the excitation coil.

Then, several arrangements of coil on $x y$ plane were also selected and simulated to find the optimum coil composition that can produce the targeted magnetic field level at the targeted position of MNPs under the excitation coil. At the end of this work, we proposed several alternative designs of excitation coil with variation in physical properties and coil arrangement based on simulation study carried out by using Ansys Maxwell. Findings from this work may contribute to the development of an excitation coil which can produce stronger and more homogeneous magnetic field generation and useful spesifically to obtain a single-sided MPI scanner suitable for human body application.

\section{RESEARCH METHOD}

The model of excitation coil and its simulation works were carried out using Ansys Maxwell to find alternative coil composition that can produce a homogeneous field with adequate strength of magnetic field sensed by the MNPs located at the varied depth of $10 \mathrm{~mm}$ to $30 \mathrm{~mm}$ under the excitation coil. Firstly, 
calculation of magnetic field generated from one available MPI system was done for comparison with the simulated result. The coil design specification data was taken from previously carried out experiment based on system shown in Figure 2 and is shown in Table 1 [9]. The excitation field generated by the excitation coil is determined by considering Bio Savart Law: magnetic field is generated when current flows through the excitation coil. To examine the magnetic field generated by the excitation coil at the targeted point where MNPs located under the excitation coil, the formula for magnetic field strength is given as in (4):

$$
B=\frac{\mu_{0} . n I}{4 L(b-a)}\left[P \ln \left\{\frac{b+\sqrt{b^{2}+P^{2}}}{a+\sqrt{a^{2}+P^{2}}}\right\}-(P-2 L) \ln \left\{\frac{b+\sqrt{b^{2}+(P-2 L)^{2}}}{a+\sqrt{a^{2}+(P-2 L)^{2}}}\right\}\right]
$$

Here, $B$ is the magnetic field strength, $\mu_{0}$ is permeability in a vacuum $\left(1.25664 \times 10^{-6}\right), n$ is number of turns, $I$ is applied current, $2 L$ is the length of coil, $a$ is the inner radius of coil, $b$ is the outer radius of coil, and $p$ is the vertical distance of the MNPs from the centre of coil. All parameters in Table 1 was inserted into (4) and the magnetic field strength, $B$ was calculated as $B_{c a l}=0.0106 \mathrm{mT}$. The calculation result will be used for comparison with the magnetic field obtained from simulation. Next, the model of the excitation coil was designed by including all parameters stated in Table 1. Cylindrical shape was choosen as coil shape because it is closely like the standard magnetic coil and stress-free design. Besides, helical shape coil with similar physical parameters was also simulated for comparison. In addition, different number of coil and different direction of current injection to the coils were included in the simulation for further analysis. Magnetic field obtained from simulation was compared with both calculation and experimental data. Note that magnetic fields measured using experimental model were 1.9, 1, and $0.6 \mathrm{mT}$ for MNPs located at 10, 20, and $30 \mathrm{~mm}$ under the excitation coil. The higher the distance of the measured point from the coil, the lower the value of magnetic field strength produced.

Table 1. Coil design specification [9]

\begin{tabular}{cc}
\hline Variable & Value \\
\hline Current & $0.8 \mathrm{~A}$ \\
Turns & 375 \\
Length of coil & $15 \mathrm{~mm}$ \\
Inner radius of coil & $10 \mathrm{~mm}$ \\
Outer radius of coil & $35 \mathrm{~mm}$ \\
Radius of wire & $0.5 \mathrm{~mm}$ \\
Free space permeability & $1.26 \mathrm{e}^{-6}$ \\
Material & Copper \\
Model & Cylinder \\
\hline
\end{tabular}

\section{RESULTS AND ANALYSIS}

The results of strength and homogeneity of magnetic field distribution generated by excitation coil that was designed and simulated using Ansys Maxwell are reported in the following subsections:

\subsection{Software testing and validation}

The coil design was set based on the parameter and value as shown in Table 1 . The 3D view of a cylindrical coil model was designed as shown in Figure 3(a). The simulation result in Figure 3(b) shows the distribution of the magnetic field generated from the cylindrical coil model to its surrounding area. It can be seen that the strength of magnetic generated becomes smaller at the point far for the coil. Red color indicates the highest value of magnetic field strength which takes place inside the excitation coil. In addition, the distribution of magnetic field strength measured across $z$ plane is at $10 \mathrm{~mm}, 20 \mathrm{~mm}$ and $30 \mathrm{~mm}$ under the excitation coil is shown in Figure 4. From simulation, magnetic field at $10 \mathrm{~mm}$ was $0.0132 \mathrm{mT}$ which is close to $B_{c a l}=0.0106 \mathrm{mT}$ that was calculated earlier. A peak value can be seen at the center of the excitation coil. It shows that simulation result was validated with the calculation result. 


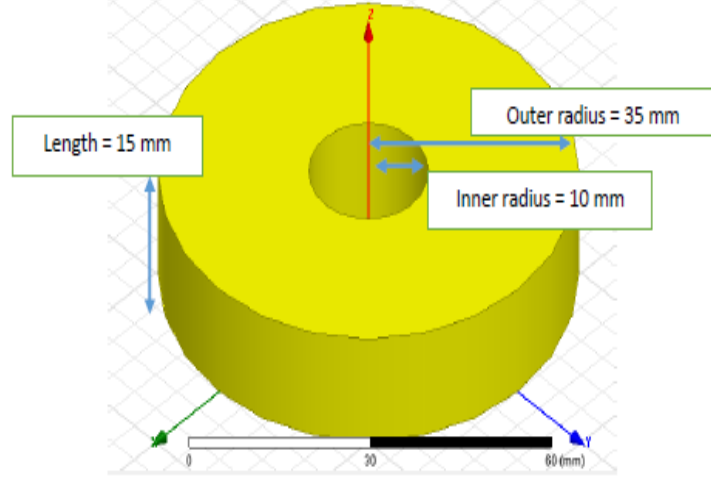

(a)

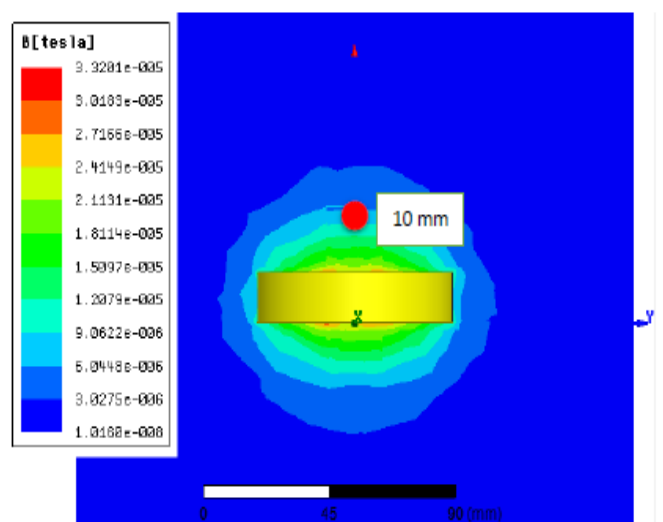

(b)

Figure 3. (a) 3D view of cylindrical coil model, and (b) Magnetic field distribution around the excitation coil on $x y z$ plane

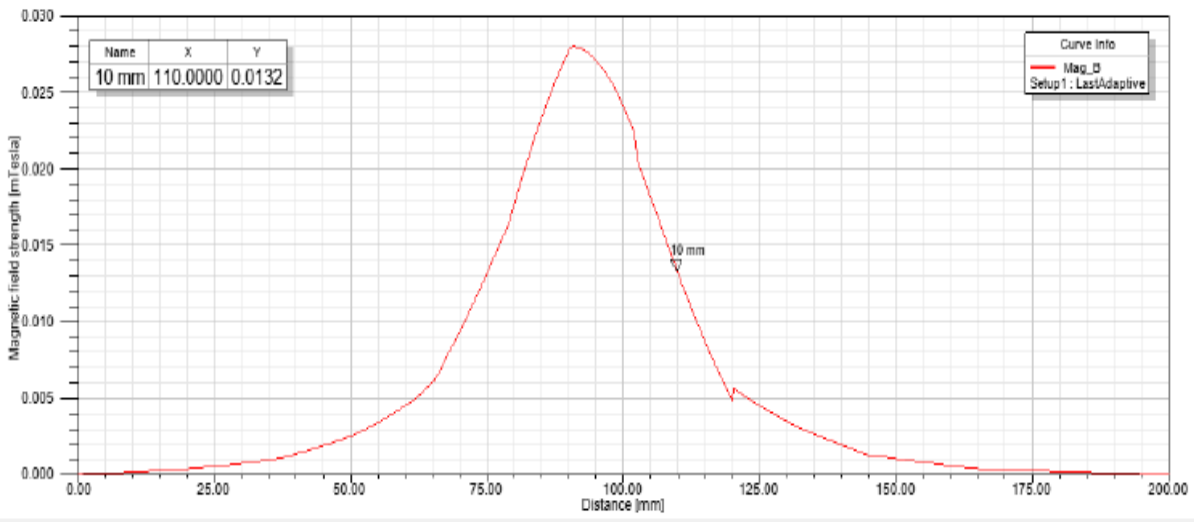

Figure 4. Magnetic field distribution across $z$ plane of the excitation coil

\subsection{Shape variation for alternative design of coil}

Next, simulation on magnetic field generated by a cylindrical coil model was done together with the helical coil model for comparison. Both models were designed based on the physical parameters in Table 1. The 3D views of both models are illustrated in Figure 5(a) and (b). As can be seen in the figure, helical coil model has gap between each turn while cylindrical model has zero gap. The gap size between the helical model turn is $1 \mathrm{~mm}$. The simulation result in Figure 6 shows the comparison between the directions of the magnetic field generated from both coils. Helical coil model produced ten times stronger magnetic field than the cylindrical model. Besides, arrows as the direction indicator of the field distribution can be used to see the homogeneity of field at each point around the coil.

Next, Figure 7 shows the magnetic field distribution across $z$ plane of the excitation coil generated by helical and cylindrical coil model. The magnetic field strengths produced are summarized in Table 2 . It clearly shows that magnetic field generated by a helical model was higher than the cylindrical model. In addition, the simulation results for both models were compared with the experimental result obtained in previous work done by Othman et. al. [9]. Magnetic fields generated from both models are still lower than the experimental one. However, we can see that the magnetic field strength by the helical model was close to the experimental data compared to cylindrical model. This finding shows the new discovery in excitation coil used in MPI system. Until now, MPI system only use cylindrical model to generate the excitation magnetic field from the coil. For future work, helical model design can be proposed as a new shape to compose an excitation coil in the MPI system. 


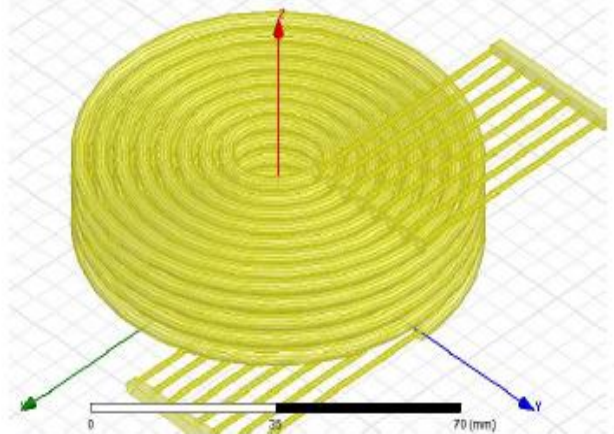

(a)

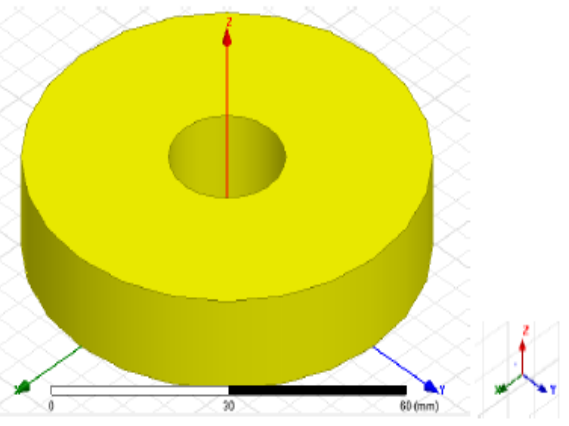

(b)

Figure 5. 3D view of, (a) Helical, (b) Cylindrical coil model

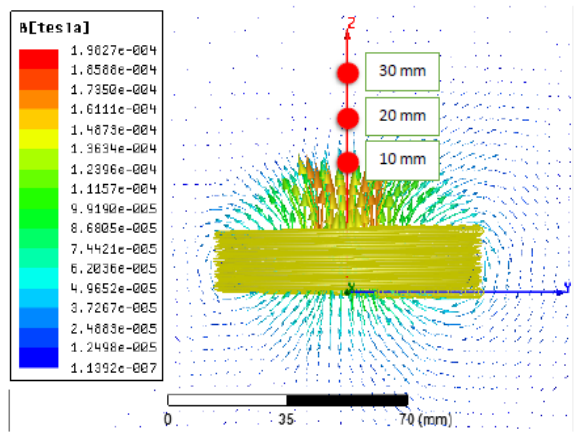

(a)

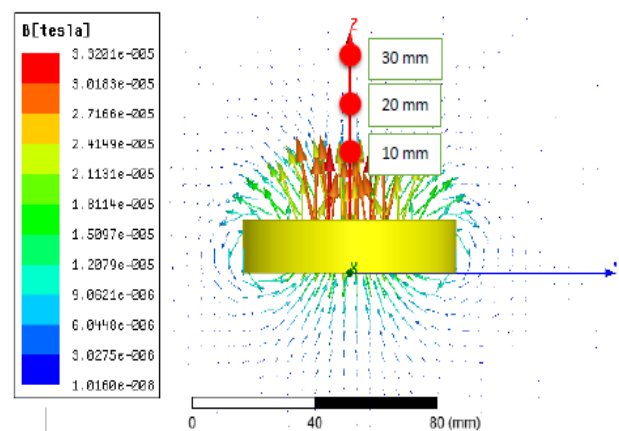

(b)

Figure 6. Magnetic field distribution with direction indicator around, (a) Helical, (b) Cylindrical coil model at $10 \mathrm{~mm}, 20 \mathrm{~mm}$ and $30 \mathrm{~mm}$ across $z$ plane of the excitation coil

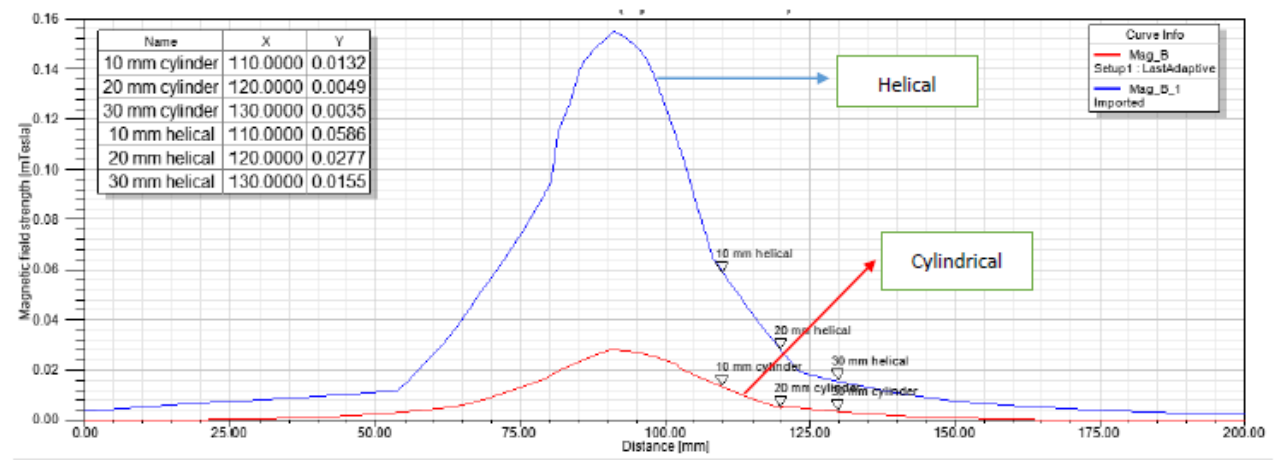

Figure 7. Magnetic field distribution across $z$ plane of the excitation coil generated by helical and cylindrical coil model

Table 2. Magnetic field strength generated by the helical, cylindrical and experiment model of coil at $10 \mathrm{~mm}$, $20 \mathrm{~mm}$ and $30 \mathrm{~mm}$ under the excitation coil

\begin{tabular}{cccc}
\hline Distance from coil $(\mathrm{mm})$ & Helical model $(\mathrm{mT})$ & Cylindrical model $(\mathrm{mT})$ & Experimental model $(\mathrm{mT})$ \\
\hline 10 & 0.0586 & 0.0132 & 1.9 \\
20 & 0.0277 & 0.0049 & 1 \\
30 & 0.0155 & 0.0035 & 0.6 \\
\hline
\end{tabular}




\subsection{Number of coils variation for alternative design of coil}

In this subsection, different numbers of cylindrical coil were simulated to see and compare the magnetic field distribution generated by the coil/s. One coil and combination of two coils were designed based on the parameter on Table 1 and were injected with similar current direction. Note that cylindrical coil model was employed regardless to the lower value of magnetic field strength generated when compared to the helix model. Two similar cylindrical coils were position adjacently on the $x y$ plane. Figure 8(a) and (b) show the magnetic field distribution with direction indicator around one and two coils. From the comparison, it shows that one coil produced stronger magnetic field and more homogeneous than two coils at $10 \mathrm{~mm}, 20$ $\mathrm{mm}$ and $30 \mathrm{~mm}$ under the excitation coil. Magnetic field strengths generated by different number of cylindrical coils and comparison with the experimental model at $10 \mathrm{~mm}, 20 \mathrm{~mm}$ and 30 under the excitation coil were summarized in Table 3 . The decreasing values in magnetic field strength with distance for both models obey the reciprocal relationship between magnetic field generated and distance from the excitation coil. Then, the mutual magnetic inductance between coil 1 and two for the case of two coils in Figure 8(b) will cancel out certain amount of magnetic field at certain point of its adjacent coil. It decreased the total magnetic field strength generated by the two coils.

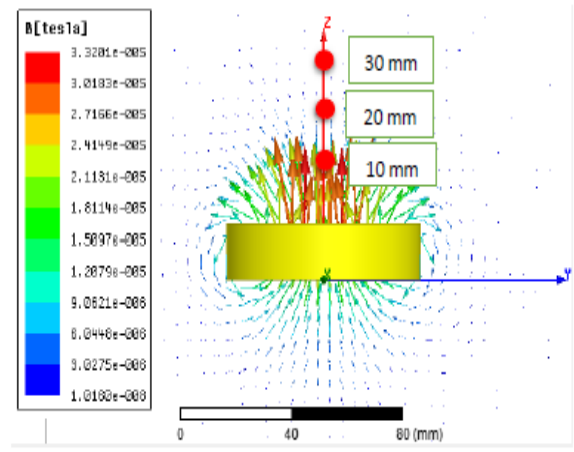

(a)
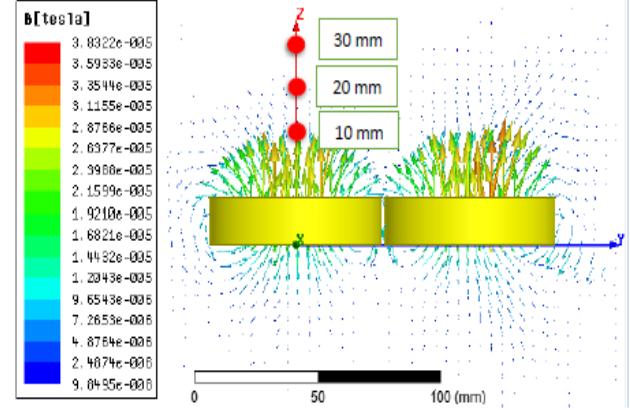

(b)

Figure 8. Magnetic field distribution with direction indicator around, (a) one, and (b) two cylindrical models positioned in parallel on $x y$ plane, at $10 \mathrm{~mm}, 20 \mathrm{~mm}$ and $30 \mathrm{~mm}$ across $z$-plane of the excitation coil

Table 3. Magnetic field strength generated by different number of cylindrical coils and experiment model of coil at $10 \mathrm{~mm}, 20 \mathrm{~mm}$ and 30 under the excitation coil

\begin{tabular}{cccc}
\hline Distance from coil $(\mathrm{mm})$ & 1 coil $(\mathrm{mT})$ & 2 coils $(\mathrm{mT})$ & Experimental model $(\mathrm{mT})$ \\
\hline 10 & 0.0132 & 0.0129 & 1.9 \\
20 & 0.0049 & 0.0053 & 1 \\
30 & 0.0035 & 0.0022 & 0.6 \\
\hline
\end{tabular}

\subsection{Current direction variation for alternative design of coil}

This subsection will discuss on the magnetic field distribution from two adjacent cylindrical coils that having different direction of current injection. Here, both models were designed based on the parameter on Table 1. Figure 9 shows the different current directions stated as (a) clockwise-clockwise, and (b) anticlockwise-clockwise direction that flow inside the two cylindrical coils. "Clockwise-clockwise" is defined for current injected into coils from similar direction while "anticlockwise-clockwise" is defined as current injected into each coil from the opposite direction of each other. The simulation results of magnetic field distribution with direction indicator of two coils with different current direction: clockwise-clockwise and anticlockwise-clockwise direction, are shown in Figure 10(a) and (b) and the related magnetic field strength at each point of $10 \mathrm{~mm}, 20 \mathrm{~mm}$, and $30 \mathrm{~mm}$ are summarized in Table 4. Coils with clockwise-clockwise current injection generated smaller magnetic field strength when compared to two coils with anticlockwise-clockwise current direction. Here, the effect of the mutual magnetic inductance between both coils can be seen to decrease the magnetic field strength produced from the "clockwise-clockwise" coil. When $I_{1}$ and $I_{2}$ flow in the similar direction following the clockwise direction in this case, magnetic field will be generated in similar direction and cancel out certain amount of magnetic field at certain point of its adjacent coil. This will decrease the magnetic field strength produced for the coils in Figure 10(a). 
Finally, the comparison between all simulated magnetic fields and experimental data as listed in Table 2, Table 3, and Table 4, shows that the magnetic fields that were generated using in this simulation work are still lower than the values measured from the real experiment. In this case, connection to resonant circuit in real measurement set up helped the excitation coil to mantain the maximum level of current to be supplied to it system. However, with the preliminary study done based on the simulation work using the Ansys Maxwell, the finding such as by replacing the cylindrical model with a helical model or by injecting currents from different direction to the two helical models may further increase the value of magnetic field produced at the targeted point.

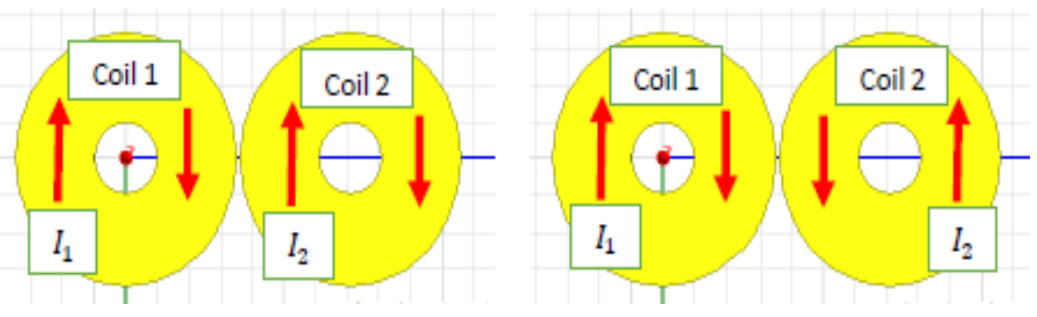

Figure 9. Different current direction, (a) clockwise-clockwise, (b) Anticlockwise-clockwise direction that flow inside the 2 cylindrical coils

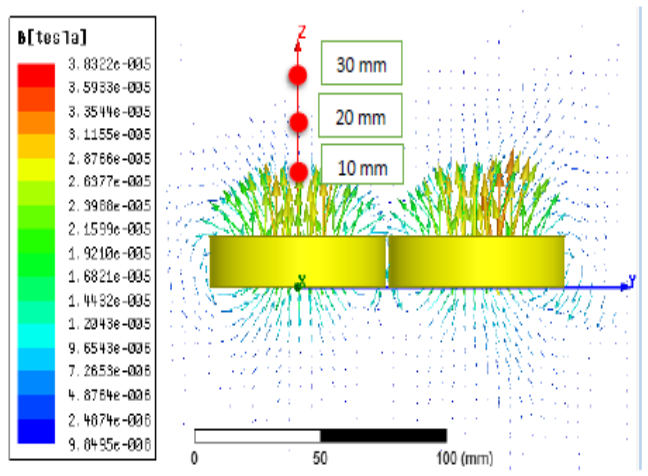

(a)

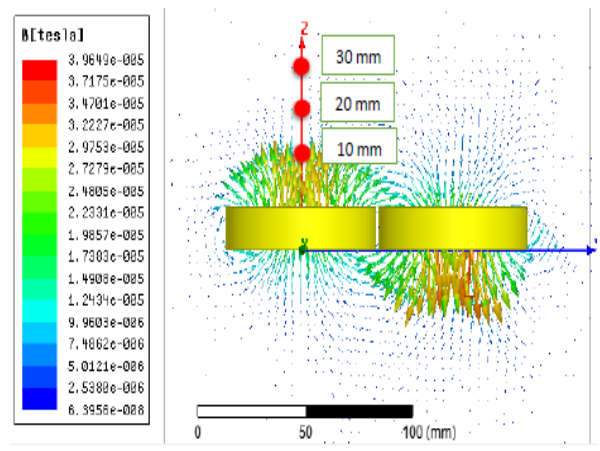

(b)

Figure 10. Magnetic field distribution with direction indicator of two coils with different current direction, (a) clockwise-clockwise, (b) Anticlockwise-clockwise direction

Table 4. Magnetic field strength generated by two coils with different current direction, (a) clockwise-clockwise, and (b) Anticlockwise-clockwise direction, at $10 \mathrm{~mm}, 20 \mathrm{~mm}$ and 30 under the excitation coil

\begin{tabular}{cccc}
\hline Distance from coil $(\mathrm{mm})$ & Clockwise direction $(\mathrm{mT})$ & Anti-clockwise and clockwise direction $(\mathrm{mT})$ & Experimental model $(\mathrm{mT})$ \\
\hline 10 & 0.0129 & 0.0145 & 1.9 \\
20 & 0.0053 & 0.0065 & 1 \\
30 & 0.0022 & 0.0028 & 0.6 \\
\hline
\end{tabular}

\section{CONCLUSION}

To sum up, a simulation study on excitation coils used in single-sided MPI scanner for human body application was done by using Ansys Maxwell. The simulated results were still far from the actual targeted magnetic field strength obtained from the experiment. However, the finding of higher magnetic field strength from a helical model compared to the cylindrical model may suggest new idea of replacing the coil shape in future MPI system. Besides, the direction of current injection also influences the value of the magnetic field generated. By injecting currents from different direction may further increase the magnetic field strength. With these findings, a more homogeneous and stronger magnetic field from excitation coil system can be obtained so that the quality of the secondary signal from the magnetized MNPs trapped under the body 
surface can be enhanced accordingly. More variation in physical properties and coil arrangement can be carried out in future to reach the targeted value of magnetic field as suggested from the real measurement using the single sided MPI.

\section{ACKNOWLEDGEMENTS}

This study is supported by MOHE under Fundamental Research Grant Scheme (FRGS) Vot 1583, Geran Penyelidikan Pascasiswazah (GPPS) Vot H308 and Universiti Tun Hussein Onn Malaysia (UTHM).

\section{REFERENCES}

[1] L. Mohammed, et al., "Magnetic Nanoparticles for Environmental and Biomedical Applications: A Review," Particuology, vol. 30, pp. 1-14, 2017.

[2] Q A Pankhurst, et al, "Progress in Applications of Magnetic Nanoparticles in Biomedicine," Journal of Physics D: Appl. Phys,.vol. 42, 2009.

[3] M. Mahmoudi and S. Laurent, "Metal Iron Oxides Series: Iron Oxide Nanoparticles for Biomedical Applications: Synthesis, Functionalization and Application," Elsevier Ltd., 2018, pp. 1-315.

[4] Harry M. Williams, "The application of magnetic nanoparticles in the treatment and monitoring of cancer and infectious diseases," Bioscience Horizons: The International Journal of Student Research, vol. 10, 2017

[5] B. Gleich and J. Weizenecker, "Tomographic Imaging Using the Nonlinear Response of Magnetic Particles," Nature, vol. 435, pp. 1214-1217, 2005.

[6] J Weizenecker, et al., "Three-dimensional real-time in vivo magnetic particle imaging," Physics in Medicine \& Biology, vol. 54, L1-L10, 2009.

[7] B. Gleich, et al., "Principles and Applications of Magnetic Particle Imaging," Springer, 2014, pp. 1-118.

[8] N. Panagiotopoulos, et al., "Magnetic Particle Imaging: Current Developments and Future Directions." International Journal of Nanomedicine, vol. 10, pp. 3097-3114, 2015.

[9] N. B. Othman, "Magnetic Nanoparticle Imaging for Biomedical Applications," PhD. Thesis, Kyushu University, 2014, pp. 1-117.

[10] T. Knopp, et al., "Magnetic particle imaging: from proof of principle to preclinical applications", Physics in Medicine \& Biology, vol. 62, no. 14, pp. R124-R178, 2017.

[11] S. Chikazumi, "Physics of Magnetism" NewYork, Wiley, 1964.

[12] T. Yoshida, N. B. Othman, T. Tsubaki, J. Takamiya and K. Enpuku, "Evaluation of Harmonic Signals for the Detection of Magnetic Nanoparticles," in IEEE Transactions on Magnetics, vol. 48, no. 11, pp. 3788-3791, Nov. 2012

[13] T. Yoshida, et al., "Characterization of Magnetically Fractionated Magnetic Nanoparticles for Magnetic Particle Imaging," Journal of Applied Physics, vol. 114, 2013.

[14] T. Yoshida et al., "Magnetic fractionation and characterization of magnetic nanoparticles for magnetic particle imaging," Jpn. J. Appl. Phys., vol. 57, pp. 080302-1- 080302-4, 2018

[15] D. Heinke et al., "Optimization of MNPs by size fractionation for MPI application," 2015 5th International Workshop on Magnetic Particle Imaging (IWMPI), Istanbul, pp. 1-1, 2015

[16] T. F. Sattel, et al., "Single-sided Device for Magnetic Particle Imaging," Journal of Physics D: Appl. Phys,.vol. 42, 2009.

[17] K. Grafe, et al., "2D Images Recorded With a Single-Sided Magnetic Particle Imaging Scanner," IEEE Transactions on Medical Imaging, vol. 35, no. 4, pp. 1056-1065, 2016 K. Gräfe, A. von Gladiss, G. Bringout, M. Ahlborg and T. M. Buzug, "2D Images Recorded With a Single-Sided Magnetic Particle Imaging Scanner," in IEEE Transactions on Medical Imaging, vol. 35, no. 4, pp. 1056-1065, April 2016.

[18] S. Tumanski, "Induction Coil Sensors-a Review," Meas. Sci. Technol., vol. 18, 2007.

[19] T. Knopp, et al., "Generation of a static magnetic field-free line using two Maxwell coil pairs," Appl. Phys. Lett., vol. 97, no. 9, pp. 092505-1-092505-3, 2010.

[20] G. Bringout and T. M. Buzug, "Coil Design for Magnetic Particle Imaging: Application for a Preclinical Scanner," in IEEE Transactions on Magnetics, vol. 51, no. 2, pp. 1-8, Feb. 2015

[21] H. Wojtczyk, et al., "Toward the Optimization of D-Shaped Coils for the Use in an Open Magnetic Particle Imaging Scanner", IEEE Transactions on Magnetics, vol. 50, no. 7, pp. 1-7, 2014.

[22] G. Bringout, et al., "A high power driving and selection field coil for an open MPI scanner," 2013 International Workshop on Magnetic Particle Imaging (IWMPI), 2013.

[23] C. Kaethner, et al., "On the way to a patient table integrated scanner system in magnetic particle imaging", Medical Imaging 2014: Biomedical Applications in Molecular, Structural, and Functional Imaging, 2014.

[24] Mason, Erica E, et al., "Design analysis of an MPI human functional brain scanner" International journal on magnetic particle imaging, vol. 3,1, 2017

[25] Ansys.com. (2017). ANSYS Maxwell: Low Frequency Electromagnetic Fields. [online] Available at: http://www.ansys.com/products/electronics/ansysmaxwell [Accessed 9 Dec. 2017] 


\section{BIOGRAPHIES OF AUTHORS}
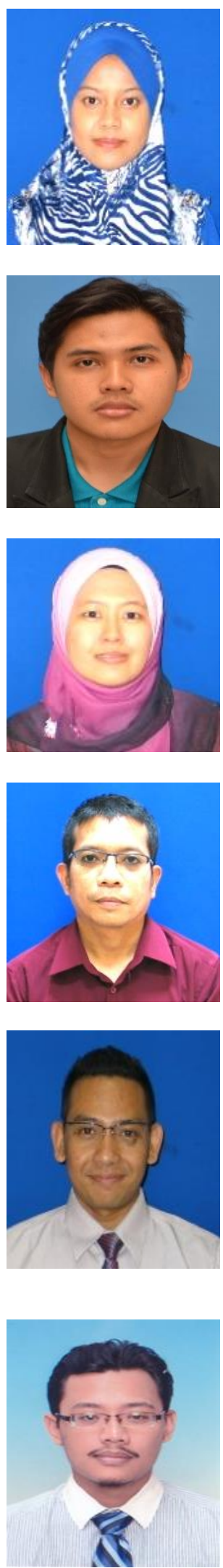

Nurmiza Othman was born in Terengganu, Malaysia, in 1983. She received the B. Sc. and M. Sc. (Electrical and Electronic Engineering) from Utsunomiya University, Japan in 2009 and $\mathrm{Ph} . \mathrm{D}$ (Electrical and Electronic Engineering) from Kyushu University, Japan in 2014. In 2007, she joined the Department of Electronic Engineering, Universiti Tun Hussein Onn Malaysia as a tutor. She has appointed as a lecturer with the same institution in 2014. Currently, she is also a Senior Researcher of UTHM Research Center for Applied Electromagnetics. She is a member of professional membership IEEE since 2014. Her research interests include Superconductor Engineering, Micro \& Nanofabrication, Nanomagnetic Materials, Magnetic Particle Imaging, and Medical Assistive Device.

Muhamad Fikri Shahkhirin Birahim was born in Johor Bahru, Malaysia in 1995. He received the Bachelor of Electronic Engineering from Universiti Tun Hussein Onn Malaysia (UTHM), Malaysia in 2018 and currently pursuing his studies in Master of Electrical Engineering at the same university. His research interest include Magnetic Particle Imaging.

Wan Nurshazwani Wan Zakaria was received B.Eng (2007) in Electronics and Mechanical Engineering from Chiba University and MSc in Mechatronics (2008) and PhD in Mechanical and System Engineering (2012) from Newcastle University. She is currently a lecturer in Faculty of Electrical and Electronic Engineering, Universiti Tun Hussein Onn Malaysia. Her current interests include Medical Robotics System specifically on development of robot force control, Image Processing and Computer Aided Diagnosis, and development of Wearable Device. She is author and co-author of several journal papers and conference proceedings.

Mohd Razali Md Tomari was born in Johor, Malaysia, in 1980. He received the B.Eng degree in Mechatronics from the Universiti Teknologi Malaysia, in 2003, M.Sc in intelligent system from the Universiti Putra Malaysia, in 2006 and $\mathrm{PhD}$ degree in computer vision and robotic from the Saitama University, Japan in 2013. In 2003, he joined Faculty of Electrical Engineering, University Tun Hussein Onn as a tutor and later on become a Senior Lecturer in 2013. His current research interest includes computer vision, pattern recognition, smart wheelchair and sensing technology.

Md Nor Ramdon Baharom was born in Kuala Lumpur, Malaysia on August 1978. He received the B.E. degree in Electrical and Electronic Engineering from School of Computing, Creative Technologies \& Engineering, Leeds Metropolitan University, United Kingdom in 2004. He received the Ph.D. degree from Electrical Energy and Power Systems Group of the School Of Electrical and Electronic Engineering at the University of Manchester in 2010. He is currently working as a lecturer in Department of Power Electrical Engineering of the Faculty of Electrical and Electronic Engineering, Universiti Tun Hussein Onn Malaysia (UTHM), Malaysia. His major research interests include high-voltage insulations, overhead-line design, lightning structure protection, electrical environmental interference impact, composite insulators and flashover of contaminated insulators.

Luqman Hakim Mahmod was born in Perak, Malaysia on October 1988. He received the B.E. in Electrical Engineering and his M.Sc. in Electrical Engineering from Universiti Tun Hussein Onn Malaysia (UTHM) in 2010 and 2012 respectively. Currently he is pursuing Ph.D. in Electrical Power Engineering from UTHM. 